\title{
Experimental Analysis of All-optical 4-ASK Signal Generation through Parametric Amplification
}

\author{
J. D. Marconi, \\ Centro de Engenharia, Modelagem e Ciências Sociais Aplicadas (CECS), Universidade Federal do ABC, Santo \\ André, 09210-170, SP, Brazil \\ Optics and Photonics Research Center at Unicamp, University of Campinas, 13083-970, Campinas, SP, Brazil. \\ M. L. F. Abbade, \\ School of Electrical Engineering, PUC-Campinas, Rod. D. Pedro I- km 136, Campinas, SP, 13086-900, Brazil. \\ A. L. A. Costa, E. Moschim, \\ School of Electrical Engineering and Computer Science, Unicamp, Av. Albert Einstein - 400, Cidade \\ Universitária Zeferino Vaz, Distrito Barão Geraldo, 13083-852, Campinas, SP, Brazil. \\ H. L. Fragnito \\ Optics and Photonics Research Center at Unicamp, University of Campinas, 13083-970, Campinas, SP, Brazil.
}

\begin{abstract}
We present experimental results concerning a new alloptical technique that multiplexes two binary signals into a quaternary one (4-ASK). The technique is based on parametric amplification. Values of the quaternary levels obtained experimentally, as a function of the extinction ratios of the binary input signals, show a rather good agreement with the simulated and the analytical results. The generated 4-ASK signals were propagated through $75 \mathrm{~km}$ of standard single-mode fiber and experienced eye penalties of only $0.5 \mathrm{~dB}$. This indicates the robustness of the technique for practical applications in metropolitan area networks.
\end{abstract}

Index Terms - Optical packets, optical signal processing, parametric amplification, quaternary amplitude-shift keying signal.

\section{INTRODUCTION}

New network applications, especially those related to video transmission (VOD, video-on-demand), have strongly increased the demand for broadband Internet [1-3]. Moreover, other new services like ultrahigh-definition video transmission, high definition TV, digital cinema, e-science, etc. $[4,5]$, which are presently in their initial stages, tend to become massively deployed in the near future. As a consequence, not only current Internet users will spend more time online, but also new users will be lured. This will certainly push the demand for network bandwidth growth in the next years. Such state of affairs strongly motivates the search for solutions that enable offering higher bandwidths without changing the current fiber infrastructure.

A very attractive solution in this direction is the development of new all-optical signal processing techniques. This would avoid the passage from the optical to the electronic domain, making possible to gain transparency of the pulse length, modulation formats, signal bit rates, etc., which as a whole will result in higher agility for the optical networks. 
One of the aims of all-optical processing is to convert one modulation format into another. For example, in [6] the conversion of non-return-to-zero to return-to-zero format is approached. In [7] and [8] all optical techniques to perform analog-to-digital and digital-to-analog all-optical processing are investigated. Conversion from on-off keying to multi-level signaling is analyzed in [9]. Also different ways of converting binary amplitude optical signals to quaternary amplitude ones are presented in [10-13]. A technique based on the parametric amplification to generate quaternary signals by coupling three lasers, two modulated with a return-to-zero (RZ) modulation format and the third one being a continuous-wave (cw) laser, was demonstrated in [12]. Recently, another technique also based on parametric amplification to generate 4-ASK signals by coupling two binary amplitude-shift keying (2ASK) signals (two lasers) was presented [15]. However, the analysis performed in [15] comprises simulation and theoretical results only.

In the present work we show, for the best of our knowledge, the first systematic analysis of experimental results concerning to the technique presented in [15], which we call parametric amplification amplitude multiplexing (PAAM). This scheme could be applied not only to enhance the bit rate of optical channels but also to perform all-optical labeling [15].

The remaining of this work is organized in the following way. Section 2 provides a brief review for the theoretical principles of parametric amplification and how it can be extended to provide PAAM. Section 3 presents our experimental and simulation setups. Results are approached in Section 4 whereas our conclusions are described in Section 5.

\section{THEORY}

Fiber optics parametric amplification is performed by coupling and launching into an optical fiber a probe signal, at frequency $f_{S}$, and a high-power un-modulated pump, at frequency $f_{P}$. In this situation, illustrated in Fig. 1(a), the fiber acts as a nonlinear medium producing a four-wave mixing interaction between the probe and the pump signals. The frequencies of the waves involved in this process satisfy the relation $2 f_{P}+f_{S}=f_{i}$, where $f_{\mathrm{i}}$ represents the frequency of a new wave called idler. When $f_{P}$ is tuned into the range of frequencies of the anomalous dispersion regime of the fiber, and the pump power is much higher than the probe signal power, such probe signal experiences a parametric gain given by

$$
G=1+x_{0}^{2}\left(\frac{\sinh x}{x}\right)^{2}
$$

where

$$
x_{0}=\gamma P_{P} L,
$$

and 


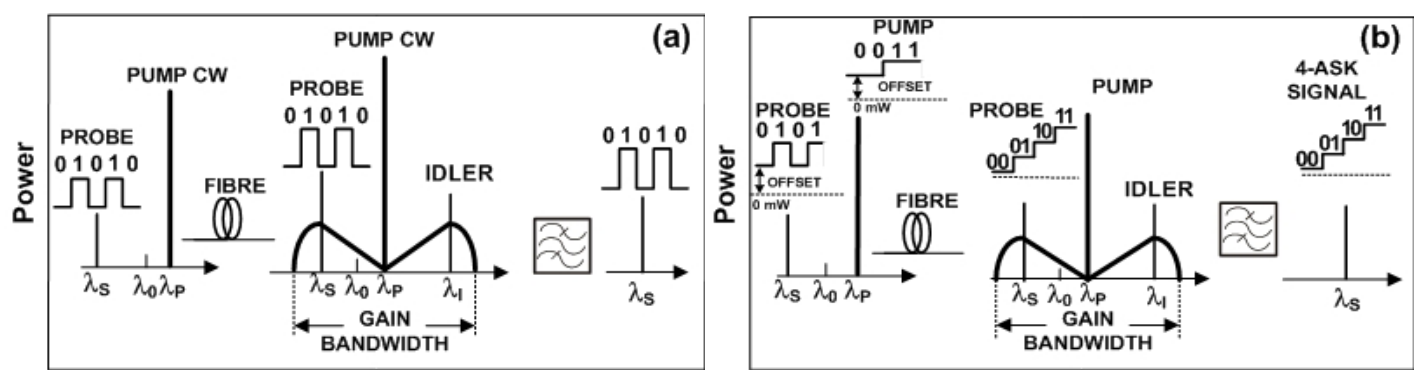

Fig. 1. Conceptual diagrams for (a) parametric amplification and (b) PAAM. $\left(\lambda_{k}=\mathrm{c} / f_{k}\right.$, where $c$ is the speed of light, $k=P$, $S, i$ represents the pump, the signal and the idler. $\lambda_{0}$ is the zero dispersion wavelength of the fiber).

$$
x=\sqrt{\left(2 \gamma P_{P}\right)^{2}-\left(2 \gamma P_{P}+\Delta \beta\right)^{2}} L / 2 .
$$

In (2) and (3) $\gamma$ is the non-linear parameter of the fiber, $P_{P}$ is the pump power (considering a lossless fiber with attenuation coefficient $\alpha=0$ ), $L$ is the fiber length, and $\Delta \beta$ is the propagation constant mismatch of the FWM process, which can be expressed as

$$
\Delta \beta=\beta_{2}\left(\omega_{P}\right)\left(\omega_{S}-\omega_{P}\right)^{2}+\frac{\beta_{4}\left(\omega_{P}\right)}{12}\left(\omega_{S}-\omega_{P}\right)^{4},
$$

with $\omega_{k}=2 \pi f_{k}(k=P, S)$, and the coefficients $\beta_{2}\left(\omega_{P}\right)$ and $\beta_{4}\left(\omega_{P}\right)$ standing, respectively, for the second and fourth order dispersion coefficients evaluated at $\omega_{P}$. Influence of fiber attenuation may be considered in (1)- (4) by simply substituting $P_{P}$ by its average value

$$
P_{P}(z=L)=P_{\mathrm{P}}(z=0)\left(1-\mathrm{e}^{-\alpha \mathrm{L}}\right) /(\alpha \mathrm{L}),
$$

where $z$ is the distance from the fiber input and $\alpha$ is the fiber attenuation coefficient.

In a first approximation, the bandwidth where the parametric gain results of practical interest can be estimated as the region limited between the two frequencies where the parametric gain is maximum. These frequencies are given by

$$
\omega_{\max , \min }=\omega_{P} \pm \sqrt{\frac{2 \gamma P_{P}}{\left|\beta_{2}\right|}},
$$

and, then, the gain bandwidth is

$$
\Delta \omega=\omega_{\max }-\omega_{\min }=2 \sqrt{\frac{2 \gamma P_{P}}{\left|\beta_{2}\right|}} .
$$

Within this region the parameter $x$ is real and the gain varies between $G_{\min }=1+x_{0}^{2}$ and $G_{\max }=1+$ $\sinh ^{2}\left(x_{0}\right)$ [16]. In order to have more precise cutoff frequencies to define the gain bandwidth, it is 
necessary to solve the transcendent equation $x^{2}=\sinh ^{2}(x)$ numerically. Outside such a bandwidth, the parametric gain decreases to values which are not suitable for practical applications.

The performance of parametric amplifiers is generally analyzed for binary probe signals with two amplitude levels representing bits "0" and "1" [17]-[20]; here, we designate the power of these levels at the fiber input, respectively, by $P_{S}^{0}(0)$ and $P_{S}^{1}(0)$, and recall that $P_{S}^{0}(0)$ is ideally null.

The technique investigated in this paper is illustrated in Fig. 1(b). Its theoretical principle is essentially the same as the one of parametric amplification, but two new features are added to the involved signals. The first is that the power of the probe signal is intentionally offset from zero, in such a way that this signal now presents an extinction ratio (ER):

$$
r_{S}=P_{S}^{1}(0) / P_{S}^{0}(0)
$$

which no longer is infinity (as in the case of ideal on-off keying signals). The second feature is that the pump is also modulated with binary information. We denote the powers, at the fiber input, of the "0" and "1" bits transmitted by the pump as $P_{P}^{0}(0)$ and $P_{P}^{1}(0)$, respectively; and assume that $P_{P}^{0}(0)$ is considerably higher than $P_{S}^{1}(0)$.

With such modifications, the modulated pump will now provide two amplification factors, $G_{P}^{0}$ or $G_{P}^{1}$, depending on whether the pump information is a " 0 " or " 1 " bit. Therefore, each of the binary levels of the signal at $f_{S}$ will experience a lower or a higher gain and will split into two new power levels at the fiber output. For instance, if the signal at $f_{S}$ is a " 1 " bit power level, the pump modulation will split it in two power levels " 01 " and " 11 " depending, respectively, if the information carried by the pump is a "0" or a " 1 " bit. The same reasoning also holds when the probe signal transports a "0" bit power level. In this situation, the probe signal at the fiber output will either assume a " 00 " or a " 10 " power level, depending again on whether pump information is a " 0 " or a " 1 " bit, respectively.

It is, thus, easy to verify that the power levels of the envelope of the signal at $f_{S}$ and at $z=L$ are

$$
\begin{aligned}
& P_{\text {out }}^{00}=G_{P}^{0} P_{S}^{0}(0), \\
& P_{\text {out }}^{01}=G_{P}^{0} P_{S}^{1}(0), \\
& P_{\text {out }}^{10}=G_{P}^{1} P_{S}^{0}(0), \\
& P_{\text {out }}^{11}=G_{P}^{1} P_{S}^{1}(0),
\end{aligned}
$$


where $P_{o u t}^{i j}$ indicates the power of the signal at $f_{S}$ and at $z=L$ when the pump and probe signal transports, respectively, bits $i$ and $j\left(i, j=\right.$ "0" or "l") at $z=0$. The amplification factors, $G_{P}^{0}$ and $G_{P}^{1}$, may be obtained from (2) and expressed by

$$
G_{P}^{0,1}=1+\left(x_{0}^{0,1}\right)^{2}\left(\frac{\sinh x^{0,1}}{x^{0,1}}\right)^{2}
$$

with

$$
x_{0}^{0,1}=\gamma P_{P}^{0,1} L
$$

and

$$
x^{0,1}=\sqrt{\left(2 \gamma P_{P}^{0,1}\right)^{2}-\left(2 \gamma P_{P}^{0,1}+\Delta \beta\right)^{2}} L / 2 .
$$

Equations (9) clearly indicate the generation of a 4-ASK signal which contains information from both the pump and probe signals. The four-levels of quaternary signals give rise to an eye-diagram structure with three eyes. In this work, we refer to the eye that involve the two lowest power levels by using the subscript "low"; analogously the subscripts "int" and "up" are utilized to refer to the eyes that involve, respectively, the two intermediate and the two higher power levels. With this convention in mind and considering the case $P_{\text {out }}^{10} \geq P_{\text {out }}^{01}\left(G_{P}^{0} P_{S}^{1}(0) \leq G_{P}^{1} P_{S}^{0}(0)\right)$, we obtain the ERs for each of such eyes as

$$
\begin{aligned}
& \rho_{\text {low }}=\frac{P_{\text {out }}^{01}}{P_{\text {out }}^{\text {ou }}}=r_{S}, \\
& \rho_{\text {int }}=\frac{P_{\text {out }}^{10}}{P_{\text {out }}^{01}}=\frac{r_{G}}{r_{S}}, \\
& \rho_{\text {up }}=\frac{P_{\text {out }}^{11}}{P_{\text {out }}^{10}}=r_{S},
\end{aligned}
$$

where $r_{G}$ is defined as the ER associated with the amplification factors, factors, $G_{P}^{0}$ or $G_{P}^{1}$,

$$
r_{G}=\frac{G_{P}^{1}}{G_{P}^{0}}
$$

Analogously, for the case $P_{\text {out }}^{10} \leq P_{\text {out }}^{01}\left(G_{P}^{0} P_{S}^{1}(0) \geq G_{P}^{1} P_{S}^{0}(0)\right)$, we obtain the ERs for each of such eyes as 


$$
\begin{gathered}
\rho_{\text {low }}=\frac{P_{\text {out }}^{10}}{P_{\text {out }}^{00}}=r_{G}, \\
\rho_{\text {int }}=\frac{P_{\text {out }}^{01}}{P_{\text {out }}^{10}}=\frac{r_{S}}{r_{G}}, \\
\rho_{\text {up }}=\frac{P_{\text {out }}^{11}}{P_{\text {out }}^{01}}=r_{G} .
\end{gathered}
$$

Equations (13) and (15) indicate that the power level distribution of the generated 4-ASK signal may be adjusted by controlling only two parameters, $r_{S}$ and $r_{G}$. Therefore, such distribution does not depend on the powers of the signal at $f_{S}$ (only on its ER), but depend on the powers utilized on the pump (equations (14), (10)-(12)). This last statement is reasonable considering that in our analytical model the power of the probe signal is much lower in comparison with that of the pump power; therefore such powers may be neglected [21]. A possible way for controlling $r_{G}$ is to set values for the pump average power and for the pump ER.

$$
r_{P}=P_{P}^{1}(0) / P_{P}^{0}(0)
$$

We note that (9), (13) and (14) hold even if $P_{P}^{0}(0)=0$, i.e. if the pump ER is infinity (ideal on-off keying). In fact, in this special case of PAAM, $G_{P}^{0}=1$ and $r_{G} \cong G_{P}^{1}$. We also note that although the theoretical model for PAAM (equations (9)-(15)) is intended to work for modulated signals, it is based on a set of equations ((1)-(5)) that is derived for $c w$ signals [21]. Therefore, a comparison between this model and experimental results is essential to verify its applicability to practical situations.

\section{EXPERIMENTAL AND SIMULATION SETUPS}

The experimental setup is shown in Fig. 2. Two DFB lasers tuned at $\lambda_{P}=1553.5 \mathrm{~nm}$ and $\lambda_{S}=$ $1552.2 \mathrm{~nm}$, were used as the pump and the signal, respectively. Here, $\lambda_{k}=c / f_{\mathrm{k}}$, with $c$ representing the speed of light in vacuum and the index $k$ standing for $P$ or $S$. The laser signals were directly modulated at $1 \mathrm{~Gb} / \mathrm{s}$ with a pseudo-random bit sequence.

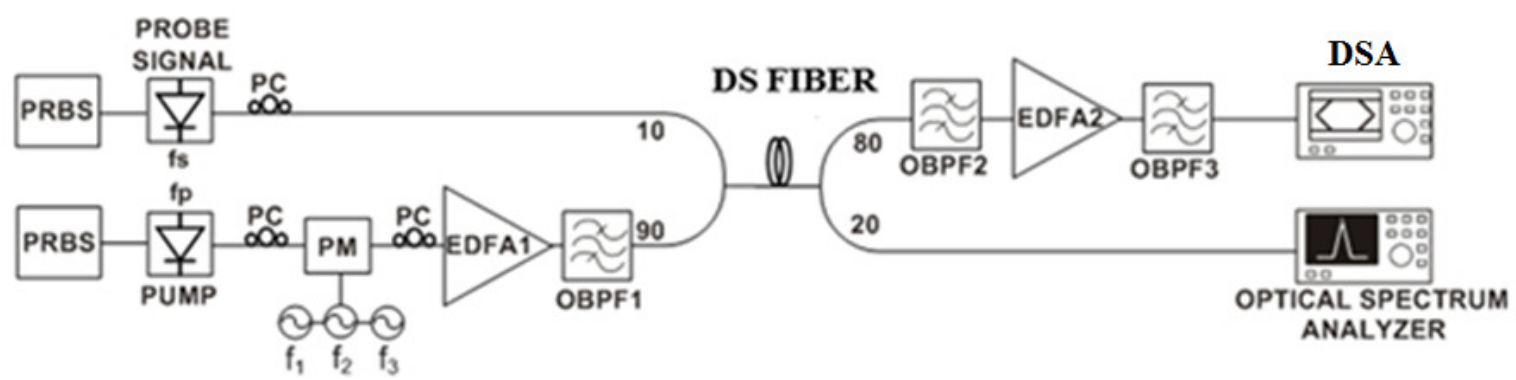

Fig. 2. Experimental and simulation setups. 
The pump linewidth was intentionally broadened by phase modulation to suppress stimulated Brillouin scattering (SBS). The phase modulator (PM) was driven with three RF signals (frequencies $f_{1}=983 \mathrm{MHz}, f_{2}=601 \mathrm{MHz}$ and $f_{3}=241 \mathrm{MHz}$ ). The pump was filtered with an optical band-pass filter (OBPF) to suppress most of the amplified spontaneous emission (ASE) from the Erbium-doped fiber amplifier (EDFA), EDFA1. The pump and the signal were finally coupled with a 90/10 coupler and launched into a dispersion shifted fiber (DSF) with length $L=7 \mathrm{~km}$, that acted as the nonlinear medium. Fiber parameters are: attenuation coefficient $\alpha=0.2 \mathrm{~dB} / \mathrm{km}$, zero-dispersion wavelength $\lambda_{0}=$ $1550.2 \mathrm{~nm}$, zero-dispersion wavelength variation $\Delta \lambda_{0}=0.08 \mathrm{~nm}$, dispersion slope $S_{0}=0.074$ $\mathrm{ps} / \mathrm{nm}^{2} / \mathrm{km}$, and nonlinear coefficient $\gamma=2.1$ (W.km) ${ }^{-1}$. Polarizations controllers (PCs) were used to align the pump and the signal states of polarization, in order to maximize the parametric gain. The average power for the pump and the signal at the fiber input were $P_{\mathrm{p}}=20.6 \mathrm{dBm}$ and $P_{\mathrm{s}}=-6.3 \mathrm{dBm}$, respectively. At the fiber output, an optical coupler delivered part of the power ( 20\%) of the optical signals to an optical spectral analyzer (OSA). The other part was transmitted through a link where the signal at $f_{S}$ was band-selected, amplified, and band-pass filtered to reduce the power of ASE accumulated in the amplification stage. The eye-diagrams of the resultant 4-ASK optical signal, whose average power was around $0 \mathrm{dBm}$, could then be observed in a digital signal analyzer (DSA).

An important goal of our work is to verify if the power levels of the 4-ASK signal may be explained by the model presented in Section 2. Among other parameters, such evaluation depends on the ERs of the binary input signals. With the equipment available in our labs, we found that we could obtain a much more accurate control of these ERs by programming the modulation index of directly modulated lasers rather than by providing an off-set current to external modulators. For this only reason, we decided to perform these proof-of-concept tests with direct modulation. Nevertheless, the analyzed technique should also hold for signals with external modulation and, because of the femtosecond response of parametric amplification [22], with higher bit rates as well. This was in fact confirmed by the simulations presented in [15].

We analyzed the transmission of this quaternary signal, through a $75 \mathrm{~km}-\mathrm{long}$ spool of standard fiber. In this case, the signal was also passed through a $-68 \mathrm{ps} / \mathrm{nm}$ dispersion compensating fiber and amplified to recover the $0 \mathrm{dBm}$ average power at the oscilloscope input.

We also performed simulations concerning the generation of the quaternary-amplitude signals. The used simulation setup is the same as the one utilized for obtaining the experimental data (Fig. 2). In addition to the experimental parameters reported above, the laser linewidth in the simulations was set to $30 \mathrm{MHz}$; this is the maximum value indicated in the manual of the utilized lasers. In order to perform simulations as similar to the experiments as possible, the phase modulation scheme used experimentally was also considered in our simulations. This point is particularly important in our case because, as it is well known, PM induces noise in the level "1" of the binary eye diagrams. As a consequence additional noise is also expected in the levels of the quaternary signals. The influence of 
$\Delta \lambda_{0}$ was accounted for by dividing the DSF in 10 segments of equal length and zero-dispersion wavelength distributed in the range from $\lambda_{0}-\Delta \lambda_{0} / 2$ to $\lambda_{0}+\Delta \lambda_{0} / 2$. The segments were randomly ordered. In each segment, fiber propagation was handled by solving the non-linear Schrödinger equation with the split-step Fourier method [23]. All simulations were performed in a commercially available software program.

\section{RESULTS AND DISCUSSION}

\section{A. Spectral analysis}

Fig. 3 presents the power spectra for the signal at (a) the fiber input, (b) the fiber output, and (c) after OBPF3. Fig. 3(b) shows that the signal at $f_{S}$ experienced a loss of $3 \mathrm{~dB}$ between the fiber output and input. As in PAAM we are interested in using the parametric interaction between the pump and probe signals for allowing the amplitude multiplexing, the absence of a parametric gain is not relevant in this experiment. The spectrum of Fig. 3(c) shows that after filtering a residual fraction of the pump remains $\sim 35 \mathrm{~dB}$ below of the generated 4-ASK signal at $f_{s}$. Such ratio could be further improved by using an additional optical band-pass filter to reduce even more the power of the spectral component at $f_{P}$.
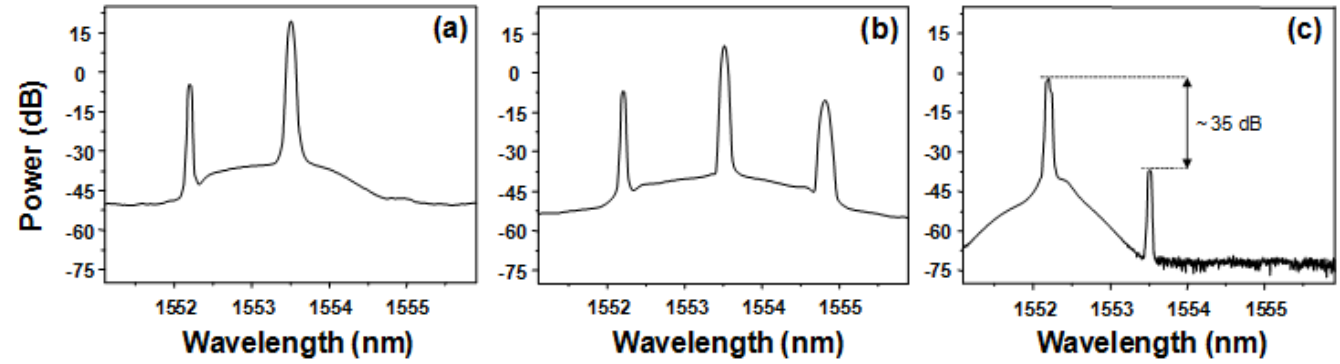

Fig. 3. Optical spectra at (a) fiber input, (b) fiber output, and (c) output of the OBPF3.

A comparison between the simulated and the experimental spectra at the fiber output is presented in Fig. 4(a). Both are very similar for powers higher than $-45 \mathrm{dBm}$. The difference between them resides in the noise region, which originates from the ASE in the pump Erbium booster amplifier. Depending on the bandwidth of the band-pass filter there will be more or less noise around the pump that will also be amplified by the parametric amplifier and cause the mentioned difference. It is observed that $f_{S}$ is placed within the region of maximum gain, where in conventional parametric amplification (pump not modulated) the signal performance is not affected by the phase modulation used to suppress the SBS [16]. However, since the pump is modulated in PAAM, the gain bandwidth attained when the pump transmits a " 1 "- bit is larger than the one reached when the pump carries a "0"- bit. For this reason, in spite of our choice for the position of $f_{s}$, we can expect that phase modulation will cause some influence on the optical signal-to-noise ratio (OSNR) performance of the quaternary signal.

It should be noted that the spectra of Fig. 4(a) is, indeed, in an average position between the real spectra achieved when the pump transmits a ' 0 '- or a ' 1 '-bit. To illustrate such spectral shift, Fig. 4(b) 
shows the simulated spectra for ' 0 '- and ' 1 '- bits, respectively $\left(r_{\mathrm{G}}=5\right)$. Obviously, such shift cannot be detected by practical OSA due to the "slow" response of this equipment.
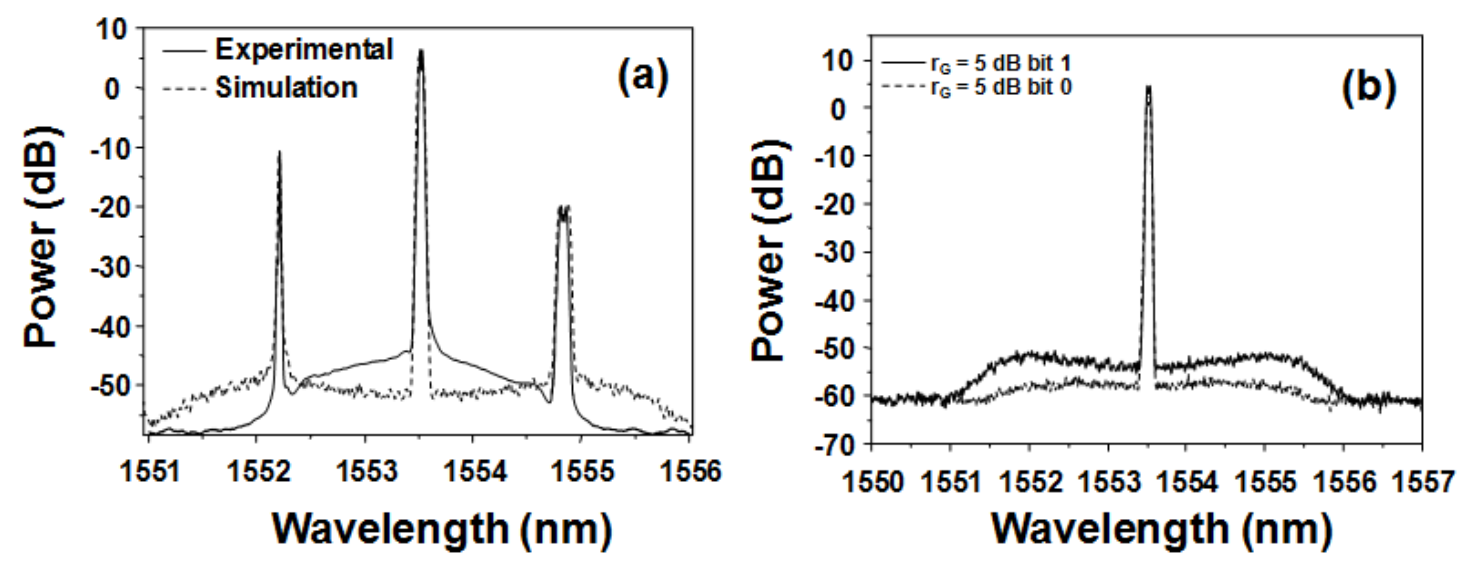

Fig. 4. (a) Experimental and simulated optical spectrum at the fiber output. (b) Spectral difference when the pump carries the bit " 1 " and the bit " 0 " ( $\left.r_{\mathrm{G}}=5\right)$.

\section{B. Time sequence comparison}

Fig. 5 illustrates two bit sequences (before amplification) carried by (a) the pump signal, (b) the probe signal, and (c) the resulting quaternary signal measured at the output of OBPF3. The pump and probe signal ERs are, respectively, $r_{P}=6.0 \mathrm{~dB}$ and $r_{S}=2.6 \mathrm{~dB}$. As expected from the considerations of Section 2, the signal of Fig. 5(c) presents four well defined power levels. It is also observed that when the bits of the pump and probe signals are repeated, the same power level of the quaternary signal is achieved. The delay between the quaternary signals obtained in the results is explained by noting that the probe signal is slightly delayed from the pump one in the experimental case (but not in the simulation case).

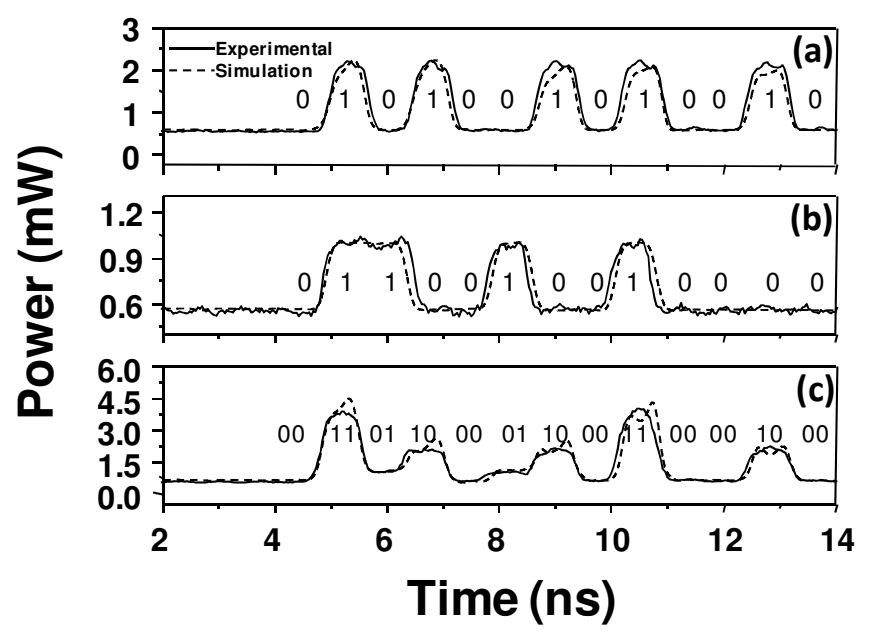

Fig. 5. Sequences: (a) binary pump, (b) binary signal before amplification, and (c) quaternary signal after amplification.

\section{ERs and comparison to the theoretical model}

Fig. 6 shows the values of $\rho_{\text {up }}, \rho_{\text {int }}$ and $\rho_{\text {low }}$ defined in (13) and (15) as a function of $r_{s}$. Each graphic corresponds to different values of $r_{G}$ that vary from 3 to $7 \mathrm{~dB}$. In all of these cases the analytical, the 
simulated, and the experimental values were plotted. The agreement is rather good in all of the cases for $\rho_{u p}$ and $\rho_{\text {int }}$. In the case of $\rho_{\text {low }}$ there exists a difference between the experimental and the analytical/ simulated values for $r_{S}>6$. One can easily see that the greater the value of $r_{G}$, the larger the difference between the experiment and the simulation; this difference is always $<1.4 \mathrm{~dB}$ and the experimental
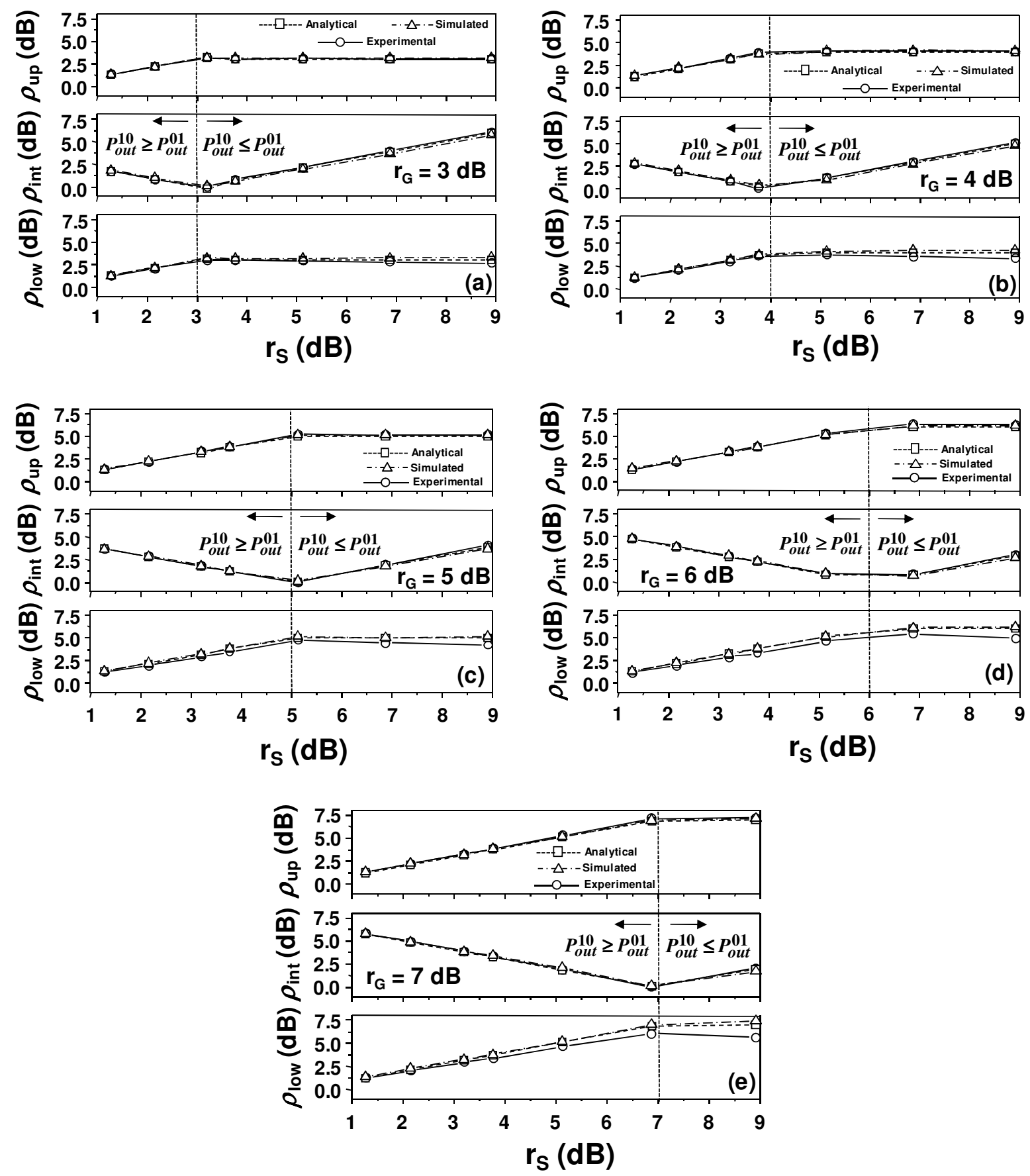

Fig. 6. Values of $\rho_{u p}, \rho_{i n t}$ and $\rho_{l o w}$ as a function of $r_{S}$ for: (a) $r_{G}=3.0 \mathrm{~dB}$, (b) $r_{G}=4.0 \mathrm{~dB}$, (c) $r_{G}=5.0 \mathrm{~dB}$, (d) $r_{G}=6.0 \mathrm{~dB}$, and (e) $r_{G}=7.0 \mathrm{~dB}$.

value is always below that of the simulated ones. Such variation may be explained by the following experimental fact. When $r_{G}$ is relatively high, the ER of the quaternary signal 


$$
\rho=\rho_{\text {low }} \rho_{\text {int }} \rho_{\text {up }}=r_{G} r_{S}
$$

will also be high. Hence, since our measurements were taken for a fixed average power, the power of level '00' moved to a relatively low value, around $\sim 10 \mathrm{dBm}$. Such power level is comparable to the one generated by the dark current of our oscilloscope. Thus, measurements for $\rho_{\text {low }}$ are not as accurate in this case as the ones for other values of $r_{G}$.

It is important to explain how the values of $r_{S}$ and $r_{G}$ were measured. To find $r_{S}$ we simply turned off the pump, transmitted a binary signal at $f_{S}$ and measured the ER of the corresponding eye-diagram at the DSA. To obtain $r_{G}$, we used a continuous-wave $(c w)$ at $f_{s}$ and a modulated pump with ER of $r_{P}$. As a result, parametric amplification provided two gains to the $c w$ signal and converted it into a binary signal whose eye diagram could be observed in the oscilloscope. The ER of such signal is precisely $r_{G}$.

\section{Eye diagrams}

Figures 7 and 8 exhibit two sets of three 4-ASK eye-diagrams for the signals at frequency $f_{s}$. The levels of the quaternary eye diagrams labeled as " 00 " and " 11 " always correspond to the upper and lower levels of the quaternary pattern, respectively. The intermediate levels may be labeled as " 01 " or "10", depending on the value of the signal extinction ratio $r_{s}$. For $r_{s}=2.2 \mathrm{~dB}$ (see Fig. 7(a)), the parametric gain obtained by signal level " 0 " when the pump level is " 1 " is higher than that obtained by signal level " 1 " when the pump level is " 0 ". Consequently, the second and third power levels of the quaternary eye diagram correspond to labels " 01 " and " 10 ", respectively. The case represented in Fig 7(b) is obtained when the parametric gain provided by pump level " 1 " to signal level " 0 " is equal to the gain provided by pump level " 0 " to probe level " 1 ". In such a situation, $r_{s} \approx \mathrm{r}_{\mathrm{G}}(3.8 \mathrm{~dB} \approx 4 \mathrm{~dB})$, so the quaternary signal degenerates into a three-level case that should be avoided.

Fig. 7(c) presents the inverse case of the one shown in Fig. 7(a). Now $r_{S}=6.8 \mathrm{~dB}>r_{G}$, and the gain provided by pump level " 1 " to signal level " 0 " is higher than that provided by pump level " 0 " to signal level "1". Consequently, the power level of the label "10" is lower than that of the power level of label " 01 ".

For the two values of $r_{G}$ presented in Figs. 7 and 8, there is a very good agreement between the eye diagrams obtained through numerical simulations and the ones measured experimentally. Similar agreements were observed for other values of $r_{G}$. 

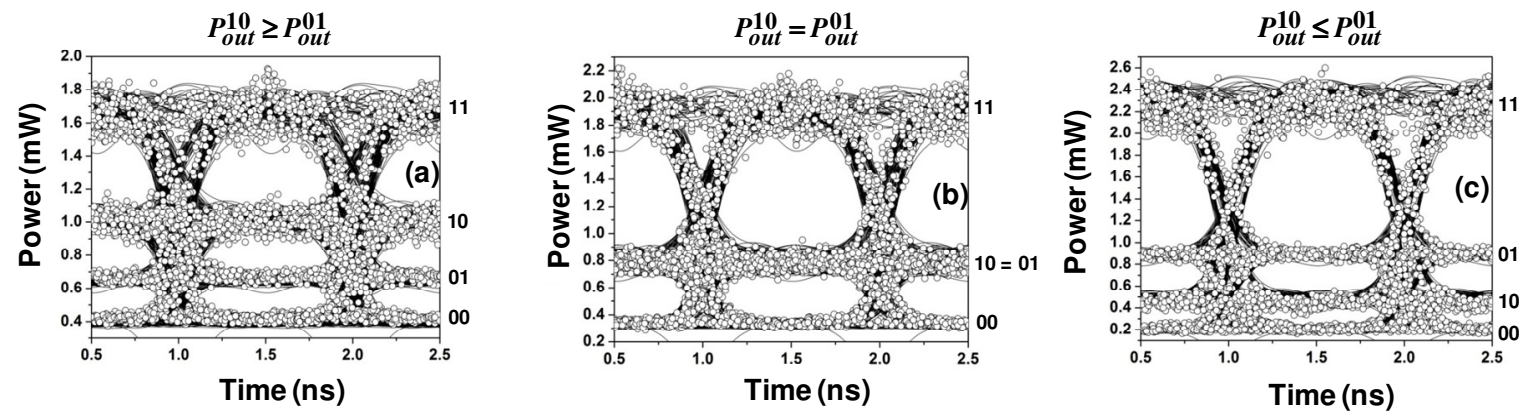

Fig. 7. Experimental (white circles) and simulated (full lines) eye diagrams for $r_{G}=4 \mathrm{~dB}$, where (a) $r_{S}=2.2 \mathrm{~dB}$, (b) $r_{S}=3.8$ $\mathrm{dB}$, and (c) $r_{S}=6.9 \mathrm{~dB}$.
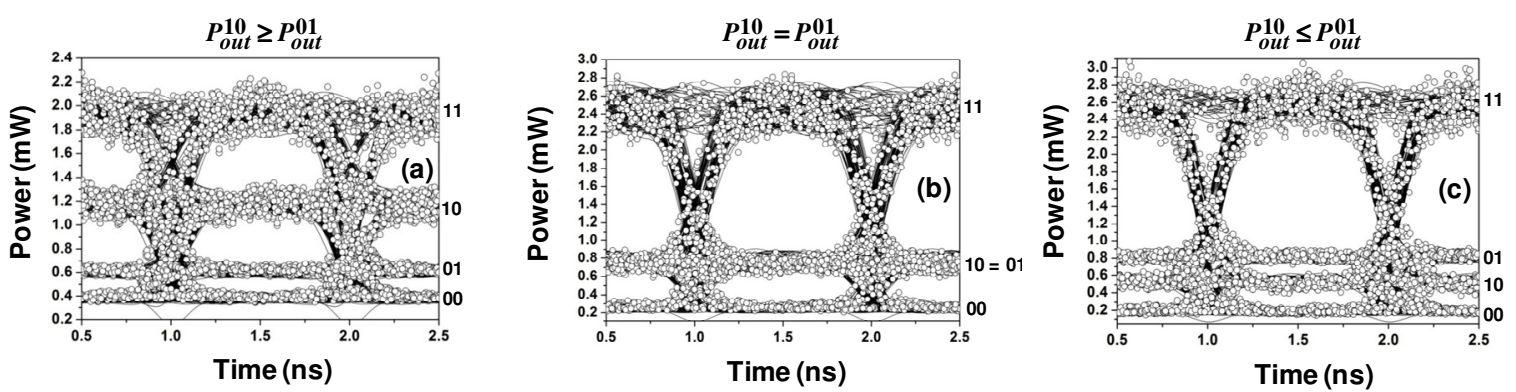

Fig. 8. Experimental (white circles) and simulated (full lines) eye diagrams for $r_{G}=5 \mathrm{~dB}:$ (a) $r_{S}=2.2 \mathrm{~dB}$, (b) $r_{S}=5.1 \mathrm{~dB}$, and (c) $r_{S}=6.9 \mathrm{~dB}$.

\section{E. Fiber propagation and BER analysis}

Finally in Fig. 9 we show the quaternary signal (a) before and (b) after propagation through $75 \mathrm{~km}$ of standard fiber. After the fiber output, an EDFA was used to raise the average power of the quaternary signal to the same value used in the fiber input $(\sim 0 \mathrm{dBm})$. As in the previous eye diagrams the agreement between experiments and numerical results is very good.
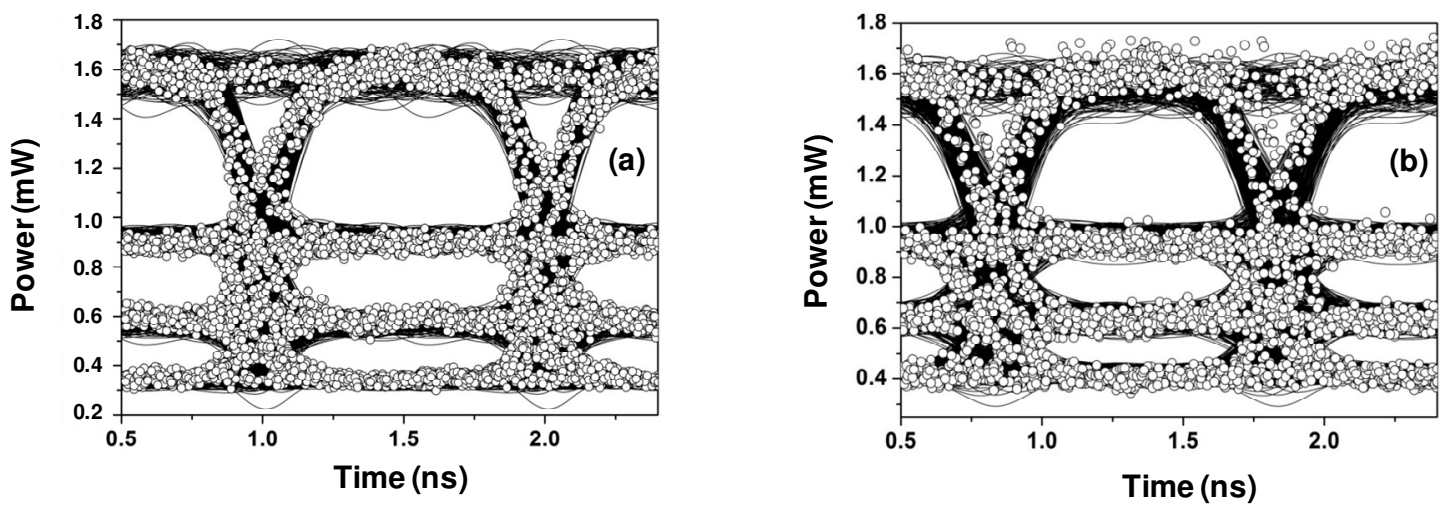

Fig. 9. Experimental (white circles) and simulated (full lines) eye diagrams: (a) before propagation, (b) after a $75 \mathrm{~km}$ standard fiber propagation with $r_{P}=0.86 \mathrm{~dB}\left(r_{G} \approx 2.5 \mathrm{~dB}\right), r_{S}=4.4 \mathrm{~dB}$.

We define the $Q$-factors for the lower, intermediate and upper eye diagrams, respectively, by 


$$
\begin{gathered}
q_{\text {low }}=\frac{i_{2}-i_{1}}{\sigma_{1}+\sigma_{2}}, \\
q_{\text {int }}=\frac{i_{3}-i_{2}}{\sigma_{2}+\sigma_{3}}, \\
q_{\text {up }}=\frac{i_{4}-i_{3}}{\sigma_{3}+\sigma_{4}},
\end{gathered}
$$

where $i_{n}$ and $\sigma_{n}$ are, respectively, the average and standard deviation of the electric current associated, after photo-detection, with the $n$-th power level of the quaternary signal. The experimental and simulation of such $Q$-factors are indicated in Table I for before and after fiber propagation.

TABLE I. Q VALUES BEFORE AND AFTER FIBER PROPAGATION.

\begin{tabular}{|c|c|c|c|c|}
\hline & \multicolumn{2}{|c|}{ Before Propagation } & \multicolumn{2}{c|}{ After Propagation } \\
\hline & Experiment & Simulation & Experiment & Simulation \\
\hline$q_{\text {low }}$ & 7.4 & 7.3 & 6.8 & 6.4 \\
\hline$q_{\text {int }}$ & 7.8 & 7.6 & 6.9 & 6.9 \\
\hline$q_{\text {up }}$ & 12.4 & 12.2 & 11.0 & 11.1 \\
\hline
\end{tabular}

With these values, we find that, after propagation, the experimental $Q$-factor penalties for the lower, intermediate and upper eyes are, respectively, of $0.4,0.5$, and, $0.5 \mathrm{~dB}$. These results, which are all lower than $1 \mathrm{~dB}$ indicate that the generated 4-ASK signal presents good robustness for propagation through lengths that are typical of metropolitan area networks.

Once $r_{G}>r_{S}$, the power levels correspond, in increasing order of magnitude, to dibits “ 00 ”, “ 01 ”, "10", and "11". We recall that, in our notation, level "ij" means that bits $i$ and $j$ were originated, respectively, from the signals at $f_{P}$ and $f_{S}$. A possible way to recover the binary information of such signals from the quaternary-amplitude signal is to convert the latter to the electronic domain and use some logic operations to interpret which bits were sent.

We consider first the logic for recovering the binary signal transmitted by $f_{P}$ from the quaternary signal of Fig. 9. Since the two lower power levels represent $i=$ "0", and the two upper power levels stand for $i=$ " 1 ", we could use a binary receiver with a threshold current between the two intermediate power levels. Therefore, assuming that noise obeys a white Gaussian noise distribution, the BER associated with such a signal may be estimated from

$$
B E R_{f p}=Q\left(q_{i n t}\right)
$$

where

$$
Q(x)=(1 / \sqrt{2 \pi}) \int_{x}^{\infty} \exp \left(-x^{2} / 2\right) d x
$$


is the complementary error function.

For recovering the binary information conveyed by $f_{S}$, we observe that the four power levels stand, in increasing order of power, $j=$ "0", " 1 ", " 0 ", " 1 " (i.e., consecutive power levels represent alternate bits). In this case, it is possible to show that the BER is the average of the BERs associated with lower, intermediate and upper eyes

$$
B E R_{f s}=1 / 3\left[Q\left(q_{\text {low }}\right)+Q\left(q_{\text {int }}\right)+Q\left(q_{\text {up }}\right)\right]
$$

Using Eq. (18) and the values of Table I, we present in Table II estimations for the BERs before and after propagation. Experimental results indicate that BERs are lower than $10^{-13}$, before transmission, and lower than $10^{-11}$, after fiber transmission. Obviously, such values could be improved by increasing the average power of the quaternary signal and/or by using optical band-pass filters with narrower bandwidths. Anyway, for the sake of comparison with other systems, it is important to relate the BERs presented in Table II with the signal-to-noise ratios of the quaternary signal. Such ratios may be estimated from the eye diagrams of Fig. 9. Before fiber propagation, the maximum BER of $8.0 \times 10^{-14}$ (for the signal at $f_{s}$ ) is associated to a $16.6 \mathrm{~dB}$ signal-to-noise ratio in the experimental case; after propagation such values are of, respectively, $7.6 \times 10^{-12}$ and $16.1 \mathrm{~dB}$. This $0.5 \mathrm{~dB}$ degradation is possibly caused by fiber dispersion penalties and to the addition of ASE noise by the EDFA used in the receiver side.

TABLE II. BER ESTIMATION BEFORE AND AFTER FIBER PROPAGATION.

\begin{tabular}{|l|c|c|c|c|}
\hline & \multicolumn{2}{|c|}{ Before Propagation } & \multicolumn{2}{c|}{ After Propagation } \\
\hline & Experiment & Simulation & Experiment & Simulation \\
\hline$B E R_{f p}$ & $1.1 \times 10^{-14}$ & $1.7 \times 10^{-14}$ & $7.4 \times 10^{-12}$ & $3.2 \times 10^{-12}$ \\
\hline$B E R_{f s}$ & $8.0 \times 10^{-14}$ & $4.8 \times 10^{-14}$ & $7.6 \times 10^{-12}$ & $2.8 \times 10^{-11}$ \\
\hline
\end{tabular}

As an alternative to this electronic detection scheme, it should be noted that the binary signals at $f_{P}$ and $f_{S}$ could also be all-optically recovered from the quaternary-amplitude signal. This could be achieved by transmitting this quaternary signal through devices that implement $S$ - and $U$-shaped transfer functions [24] or by using other techniques [7] as well.

\section{F. Discussion on optimal power level distribution}

The optimal power level distribution for 4-ASK signals depends on the dominant kind of noise and was analyzed in [25]. If thermal noise is dominant, the power levels of such signal should be uniformly distributed, i.e. in proportions of 0:1:2:3; this implies an infinite $\rho_{\text {low }}, \rho_{\text {int }}=2.0$, and $\rho_{u p}=1.5$. In case ASE noise is dominant then a quadratic, 0:1:4:9, power level spacing is required, which leads to $\rho_{\text {int }}=4.0, \rho_{\text {up }}=2.25$ and, again, to an infinite $\rho_{\text {low }}$. Equations (13) and (15) show that, regardless of the dominant kind of noise, $r_{S}$ and $r_{G}$ may be easily set to provide any optimal values of $\rho_{\text {int }}$ and $\rho_{u p}$. 


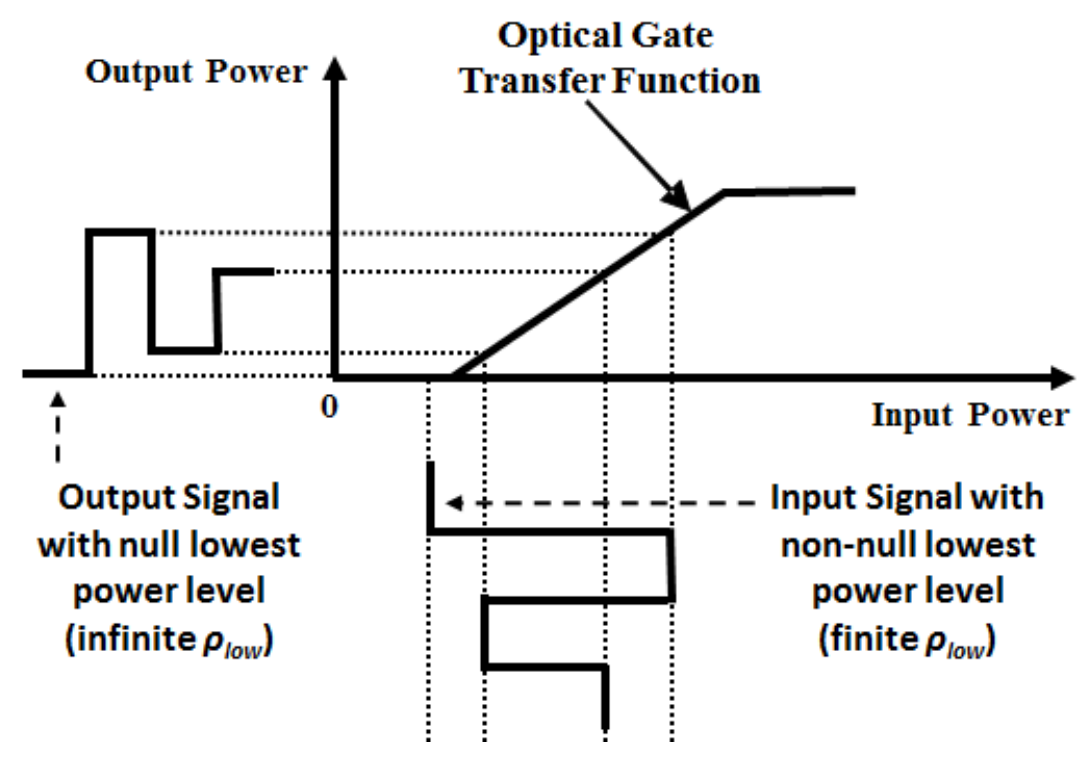

Fig. 10. Transmission of the quaternary signal generated by PAAM through an optical gate.

For instance, in the case described by (15) and dominant thermal noise, one could set $r_{G}=1.5(1.7 \mathrm{~dB})$ and $r_{s}=3.0(4.7 \mathrm{~dB})$ to achieve the previously mentioned optimal values. Similarly, for dominant ASE noise, such values should be adjusted to $r_{G}=2.25(3.5 \mathrm{~dB})$ and $r_{s}=9.0(9.5 \mathrm{~dB})$. However, (13) and (15) also show that the investigated technique imposes the restriction that $\rho_{\text {low }} \rho_{\text {up }}$; therefore, PAAM is not able to provide the optimal value for $\rho_{\text {low }}$.

A possible all-optical approach to solve this problem is to transmit the 4-ASK signal generated by PAAM through an optical gate whose ideal transfer function is illustrated in Fig. 10. If the power levels of the quaternary signal are adjusted to fit the transfer function as also indicated in Fig. 10, the values of $\rho_{\text {int }}$ and $\rho_{\text {up }}$ are unchanged by the optical gate. On the other hand, the power of the lowest level of the input 4-ASK signal is mapped to a null power value. In this way $\rho_{\text {low }}$ achieves the optimal (infinite) value and the optimal power level distribution is obtained.

In practice, optical gates are implemented by using, for example, one or two stages of FWM [26], and their transfer functions do not exhibit a linear behavior such as the one presented in Fig. 10. Anyway, the discussion above remains valid because it is possible to pre-distort the power levels of the quaternary signal generated by PAAM in such a way that the optimal values of $\rho_{\text {int }}$ and $\rho_{\text {up }}$ are achieved when this signal is transmitted through the optical gate.

Finally, we note that in our experiments we found that the minimum BER after the considered propagation occurred for $r_{G}=2.5 \mathrm{~dB}$ and $r_{s}=4.4 \mathrm{~dB}$ (Fig. 9). These values are close to but do not match the above mentioned values for the dominance of thermal noise. Besides of a finite $\rho_{\text {low }}$, this difference is also influenced by the contributions of ASE noise and of the noise induced by the pump phase modulation utilized to prevent SBS. 


\section{CONCLUSION}

For the best of our knowledge, we have presented the first systematic experimental analysis concerning PAAM. It was shown that our theoretical model presents a very good agreement (difference lower than $1.4 \mathrm{~dB}$ ) with experimental results. This is interesting because such model, (9)(15), considers a modulated pump but it is based on equations (1)-(5) derived for $c w$ signals. Therefore, our results also indicate that the conventional equations used for describing parametric amplification ((1)-(5)) may also hold for modulated signals.

We also observed a good agreement between simulation and experimental results, even for time and propagation analysis where noise contributions are clearly relevant. Such agreement was possible because our simulations took into account the pump linewidth enhancement caused by the phase modulator and also the random variation on the fiber zero-dispersion wavelength.

Our experiment also shows that the 4-ASK signal generated by PAAM may present BERs as low as $7.6 \times 10^{-12}$ even after propagation through a $75-\mathrm{km}$ long standard fiber. Such value was obtained after searching for an optimal power level distribution, but it could be certainly improved by utilizing narrower filters or quaternary signals with higher average powers. The good experimental BER performance of PAAM reinforce that it could be used in practical applications, at least where length scales are comparable to the ones of metropolitan area networks (MANs). MANs are, in fact, the kind of network where PAAM labelling application is relevant [15] and where the simplicity of detecting intensity-modulated signals is still advantageous over phase-modulated ones [27].

\section{ACKNOWLEDGMENT}

The authors thank FAPESP and CNPq for funding this work under processes 574017/2008-9, 309031/2008-7, 142270/2007-5 and 2008/5787-2. The authors also thank to VPI Photonics Inc. for providing us academic licenses of VPItransmissionMaker 8.5.

\section{REFERENCES}

[1] E. Desurvire, "Capacity demand and technology challenges for lightwave systems in the nest two decades," J. Lightwave Technol., vol. 24, no. 12., pp. 4697-4710, Dec. 2006.

[2] J. Berthold, A. A. M. Saleh, L. Blair, and J. M. Simmons, "Optical networking: past, present, and future," J. Lightwave Technol., vol. 26, no. 9, pp. 1104-1118, May 2008.

[3] A. E. Willner, O. F. Yilmaz, J. Wang, X. Wu, A. Bogoni, L. Zhang, and S. R. Nuccio, "Optically efficient nonlinear signal processing,", "IEEE J. Sel. Top. Quantum Electron., in press.

[4] C. E. Abosi, R. Nejabati, and D. Simeonidou, "A novel service composition mechanism for the future optical internet," J. Opt. Commun. Netw., vol. 1, no.2, pp. A106- A120, Jul. 2009. 
[5] S. Namiki, T. Kurosu, K. Tanizawa, J. Kurumida, T. Hasama, H. Ishikawa, T. Nakatogawa, M. Nakamura, and K. Oyamada, "Ultrahigh-definition video transmission and extremely green optical networks for future," IEEE J. Sel. Top. Quantum Electron., in press.

[6] J. Wang, J. Sun, Q. Sun, D. Wang, M. Zhou, X. Zhang, D. Huang, and M.M. Fejer, "Experimental observation of all-optical non-return to zero-to-return-to-zero format conversion based on cascaded second-order nonlinearity assisted by active mode-locking," Opt. Lett., vol. 32. no. 16, pp. 2462-2464, Aug. 2007.

[7] S. Oda and A. Maruta, "Two-bit all-optical analog to-digital conversion by filtering broadened and split spectrum induced by soliton effect or self-phase modulation in fiber," IEEE J. Sel. Top. Quantum Electron., vol. 12, no. 2, pp. 307-314, May 2006.

[8] T. Nishitani, T. Konishi, H. Furukawa, and K. Itoh, "All-optical digital-to-analog conversion using pulse pattern recognition based on optical correlation processing," Opt. Express., vol. 13. no. 25. pp.10310-10315, Dec. 2005.

[9] K. Mishina, S. Kitagawa, and A. Maruta, "All-optical modulation format conversion from on-offkeying to multiple-level phase-shift-keying based on nonlinearity in optical fiber," Opt. Express, vol. 15, no. 13, pp. 8444-8453, Jun. 2007.

[10] H. Soto and A. Gutiérrez, "All-optical 2-to-4 level encoder based on cross polarization modulation in a semiconductor optical amplifier utilized to develop an all-optical 2 input digital multiplexer,” Opt. Express, vol. 14, no. 20, pp. 9000-9005, Sep. 2006.

[11] M. L. F. Abbade, E. A. M. Fagotto, R. S. Braga, I. E. Fonseca, E. Moschim, and F.R. Barbosa, "Optical amplitude multiplexing through four-wave mixing in optical fibers," IEEE Photon. Technol. Lett., vol. 17, no. 1, pp. 151-153, Jan. 2005.

[12] Porzi, C.; Nguyen, A.T.; Poti, L.; Bogoni, A., "Binary-to-Quaternary ASK Encoding in the Optical Domain with Semiconductor Optical Amplifiers," IEEE Photon. Technol. Lett., vol.21, no.10, pp.654-656, May 2009.

[13] G. T. Zhou, K. Xu, J. Wu, C. Yan, Y. Su, and J. T. Lin, "Self-pumping wavelength conversion for DPSK signals and DQPSK generation through four-wave mixing in highly nonlinear optical fiber,” IEEE Photon. Technol. Lett., vol. 18, no. 22, pp. 2389-2391, Nov. 2006.

[14] Y. Liang, P. C. Chui, and K. K. Y. Wong, "Demonstration of an all-optical 2-to-4 level encoder based on an optical parametric amplifier," Res. Lett. Opt., vol. 2009, Article ID 981620, Jan. 2009.

[15] M. L. F. Abbade, A. L. A. Costa, F. R. Barbosa, F. R. Durand, J. D. Marconi, and E. Moschim, "Optical Amplitude Multiplexing through Parametric Amplification in Optical Fibers," Opt.Comm., vol. 283, no. 3, pp. 454-463, Feb. 2010.

[16] J. M. Chavez Boggio, A. Guimarães, F. A. Callegari, J. D. Marconi, and H. L. Fragnito, “Q penalties due to pump phase modulation and pump RIN in fiber optic parametric amplifiers with nonuniform dispersion," Opt.Comm., vol. 249, no. 4-6, pp. 451-472, May. 2005. 
[17] G. Kalogerakis, K. Shimizu, M. E. Marhic, K. K. Y. Wong, K. Uesaka, and L. G. Kazovsky, "High-repetition-rate pulsed-pumped fiber OPA for amplification of communication signals," ," J. Lightwave Technol., vol. 24, no. 8, pp. 3021-3027, Aug. 2006.

[18] A. Mussot, E. Lantz, A. Durécu-Legrand, C. Simonneau, D. Bayart, H. Maillotte, and T. Sylvestre, "Simple method for crosstalk reduction in fiber optical parametric amplifiers," Opt.Comm., vol. 275, no. 2, pp. 448-452, Jul. 2007.

[19] J. M. Chavez Boggio, , F. A. Callegari, J. D. Marconi, A. Guimarães and H. L. Fragnito, "Influence of zerodispersion wavelength varaiations on cross-talk in single-pumped fiber optic parametric amplifiers," Opt.Comm., vol. 242, no. 4-6, pp. 471-478, Dec. 2004.

[20] C. Peucheret, M. Lorenzen, J. Seoane, D. Noordegraaf, C. V. Nielsen, L. Gruner-Nielsen, and K. Rottwitt, "Amplitude regeneration of RZ-DPSK signals in single-pumped fier-optic parametric amplifer,", "IEEE Photon. Technol. Lett., vol. 21, no. 13, pp. 872-874, Jul. 2009.

[21] S. Song, C. T. Allen, K. R. Demarest, and R. Hui, "Intensity-dependent phase-matching effects on four-wave mixing in optical fibers," J. Lightwave Technol., vol. 17, no. 11, pp. 2285-2290, Nov. 1999.

[22] A. B. Grudinin, E. M. Dianov, D. V. Korobkin, A. M. Prokhorov, V. N. Serkin, and D. V. Khaidarov, "Decay of femtosecond pulses in single-mode optical fibers," JETP Lett., vol. 46, no. 11, pp. 221-225, Sep. 1987.

[23] G. P. Agrawal, Nonlinear Fiber Optics, 3rd ed. New York: Academic Press, 2001.

[24] E.A. M. Fagotto and M. L. F. Abbade, "All-optical demultiplexing of 4-ASK optical signals with four-wave mixing optical gates," Opt.Comm., vol. 283, no. 6, pp. 1102-1109, Mar. 2010.

[25] S. Walklin, J. Conradi, "Multilevel signaling for increasing the reach of $10 \mathrm{~Gb} / \mathrm{s}$ lightwave systems," Lightwave Technology, Journal of , vol.17, no.11, pp.2235-2248, Nov 1999.

[26] A. Bogris, D. Syvridis, "Regenerative properties of a pump-modulated four-wave mixing scheme in dispersion-shifted fibers," Lightwave Technology, Journal of , vol.21, no.9, pp. 1892- 1902, Sept. 2003.

[27] Javier Cano Adalid, "Modulation Format Conversion in Future Optical Networks," Master Thesis, Technical University of Denmark, March 2009. 